\title{
Percepción Intraoperatoria del Paciente sobre la Preparación de la Cavidad de Acceso Endodóntico
}

\author{
Patient Intraoperatory Perception About Endodontic Access Cavity Preparation
}

Paris, Leslie'; Figueroa, Daniela1'; Alcántara, Raúl2; Lagos, Germán³; Muñoz, Francisca²

\begin{abstract}
PARIS, L.; FIGUEROA, D.; ALCÁNTARA, R.; LAGOS, G. \& MUÑOZ, F. Percepción intraoperatoria del paciente sobre la preparación de la cavidad de acceso endodontico. Int. J. Odontostomat., 12(3):211-218, 2018.

RESUMEN: La creencia de que el tratamiento de endodoncia es el tratamiento dental más doloroso es algo habitual. A pesar de ello, la percepción intraoperatoria durante el tratamiento de endodoncia ha sido poco estudiada. Por ello, el objetivo de esta investigación fue describir la percepción de dolor y la experiencia intraoperatoria del paciente tratado en endodoncia, durante la etapa de acceso endodóntico. Se analizaron las valoraciones sobre dolor intraoperatorio realizadas por 20 pacientes, tratados en la clínica de especialidad de una universidad tradicional chilena, durante la etapa de acceso endodóntico en molares. Para ello se utilizaron escalas cuantitativas de valoración de la intensidad del dolor y el análisis de contenido cualitativo de una entrevista semi-estructurada. Se observó que los participantes de sexo femenino, los tratamientos en molares mandibulares y en dientes con diagnóstico de pulpitis irreversible sintomática obtuvieron medias mayores en cuanto a la valoración de la intensidad del dolor. Sin embargo, en la mayoría de los casos el dolor fue descrito como leve. En el análisis cualitativo lo descrito por los participantes se agrupó en 10 categorías. Se observó un mayor porcentaje de referencias a la categoría "Ansiedad ante el tratamiento" (16 \%) seguido de "Percepción de la atención profesional" (14 $\%)$. Sin embargo, también destacaron las referencias al dolor en su totalidad (25\%) ya sea a "Ausencia de dolor" (13\%) o a "Presencia de dolor en algún grado" (12\%). Se concluyó que entre los participantes de esta investigación existió percepción de dolor intraoperatorio durante la etapa de acceso endodóntico, sin embargo, este fue de carácter leve en la mayoría de los casos.
\end{abstract}

PALABRAS CLAVE: dolor, intraoperatorio, endodoncia, estudio mixto.

\section{INTRODUCCIÓN}

En la práctica clínica, el odontólogo tiene que lidiar con frecuencia, la percepción pública de que el tratamiento de endodoncia es el tratamiento dental más doloroso (Thomas, 2015). A raíz de ello, numerosos pacientes se enfrentan a este tratamiento con una carga emocional adicional, provocada por la expectativa de dolor a soportar (Segura-Egea \& Cisneros-Cabello, 2008). Sin embargo, en un estudio realizado en Estados Unidos, sólo el $17 \%$ de los sujetos que experimentaron un tratamiento de endodoncia lo describieron como su experiencia dental más dolorosa (Pak \& White, 2011). Investigaciones que han estudiado la percepción de dolor durante el tratamiento de endodoncia, indican que a menudo es menor a lo esperado y, además, sugieren que cada terapia endodóntica es percibida como una nueva experiencia (Watkins et al., 2002). La literatura afirma que la percepción de dolor leve, o molestias durante la intervención, se relacionan con la falta de utilización de las posibilidades que la anestesia local ofrece y/o su refuerzo. Por esto, hoy en día, la equiparación entre endodoncia y dolor es inadecuada (Segura-Egea \& Cisneros-Cabello).

Conseguir eliminar el dolor del paciente, aporta al endodoncista una de las mayores satisfacciones personales y profesionales, además de fortalecer la confianza, el compromiso y el reconocimiento a su labor. El paciente endodóntico, en muchos casos afectado por pulpitis o periodontitis sintomáticas con sensibili-

\footnotetext{
${ }^{1}$ Programa de Especialización en Endodoncia 2017, Facultad de Odontología, Universidad de Concepción, Concepción, Chile.

${ }^{2}$ Departamento de Odontología Restauradora, Facultad de Odontología, Universidad de Concepción, Concepción, Chile.

${ }^{3}$ Facultad de Ciencias Sociales, Universidad de Concepción, Concepción, Chile.
} 
zación central y periférica, representa un desafío para el control del dolor, lo que dificulta el actuar del profesional (Woolf \& Salter, 2000). El dolor intraoperatorio durante el tratamiento endodóntico puede tener múltiples agentes causales, por ejemplo: variaciones anatómicas, errores técnicos en la administración de anestésicos, soluciones defectuosas, la ansiedad del paciente y/o la inflamación (Kayaoglu et al., 2016). Además, los cambios morfogenéticos provocados por la inflamación neurogénica pueden hacer a las fibras nerviosas muy resistentes a la anestesia (Segura-Egea $\&$ Cisneros-Cabello). Incluso, se ha postulado que la aprehensión del paciente, junto con los efectos de la inflamación tisular, producen una baja en el umbral del dolor, situación que disminuye el efecto del anestésico (Walton \& Torabinejad, 1992).

A pesar de la importancia que tiene el conocer más sobre el dolor, su estudio es complejo debido a que su percepción es subjetiva. Es decir, sólo el paciente conoce su dolor e intensidad, aspectos que varían mucho de acuerdo a antecedentes individuales (Thomas). Por ende, se debe considerar el dolor como un estado emocional y no solamente una sensación primaria, como la visión o la audición, lo que hace difícil determinar con precisión el grado del mismo y su valoración debe atender necesariamente al informe del enfermo (Serrano-Atero et al., 2002). Asimismo, los factores psicosociales son importantes en la experiencia del dolor agudo y crónico orofacial; dentro de ellos, el miedo al dolor es un importante determinante emocional que implica la aprehensión irracional, hacia la nocicepción resultante de cualquier fuente (Randall et al., 2016). Es reconocido que el miedo al dentista, al tratamiento, la ansiedad, la aprensión y, posiblemente, otros factores psicológicos influyen en los umbrales de percepción del dolor y de reacción del paciente (Siqueira Jr. \& Barnett, 2004).

Pese a lo anterior, la percepción intraoperatoria durante el tratamiento de endodoncia ha sido poco estudiada, tanto a nivel mundial como nacional. Dentro de las ventajas de conocer mayores antecedentes al respecto, se encuentra la posibilidad de comunicar previamente la eventual presencia de dolor durante tratamiento y sus características, lo que aumentaría la confianza del paciente con el operador. Además, permitiría tomar medidas preventivas, tales como administrar medicamentos preoperatorios para aumentar la eficacia anestésica, aumentar el volumen de la solución anestésica, seleccionar una solución anestésica más eficaz o administrar anestesia suplementaria por adelantado (Kayaoglu et al.). A raíz de lo anterior, el objetivo de esta investigación fue, describir la percepción de dolor y la experiencia intraoperatoria del paciente tratado en endodoncia, durante la etapa de acceso endodóntico.

\section{MATERIAL Y MÉTODO}

Se realizó un estudio descriptivo, de corte transversal, con una estrategia teórico-metodológica mixta. Los participantes fueron seleccionados mediante un muestreo por conveniencia, mientras realizaban su tratamiento en la clínica de especialidad de Endodoncia. La participación de los pacientes, estuvo mediada por un proceso de consentimiento informado y el proyecto de investigación fue aprobado por parte del comité de la institución donde se realizó el estudio (C.I.Y.B Nº 0917). Fueron incluidos en la muestra, pacientes mayores de 18 años, que requirieron tratamiento endodóntico de molares con desarrollo radicular completo. Se excluyeron pacientes en condición de embarazo; con capacidad de comunicación limitada; que ingirieron AINES en las 24 horas previas a la intervención; bajo tratamiento o medicación de tipo siquiátrico; que por su condición sistémica requerían tratamiento bajo anestesia sin vasoconstrictor; diagnóstico pulpar de diente previamente tratado y diente con terapia previamente iniciada; con diagnóstico apical de absceso apical agudo; con destrucción coronaria que incluía cámara pulpar ya sea por caries y/o fracturas; y con antecedentes de traumatismo dentoalveolar.

Previo al procedimiento, se realizó un examen clínico y se completó una ficha para cada paciente registrando su edad, sexo, diente a tratar y diagnóstico. El acto operatorio consistió en conformar una cavidad de acceso endodóntico, utilizando anestesia local infiltrativa para la arcada superior y troncular al nervio dentario inferior en la arcada mandibular. Se utilizó un tubo de anestesia de mepivacaina al $2 \%$ con vasoconstrictor (epinefrina 1:100.000). En caso de manifestar dolor durante el procedimiento, la anestesia fue reforzada utilizando otro tubo de la misma solución, lo que también fue registrado. La cavidad de acceso se conformó bajo aislamiento absoluto, posterior a la eliminación de caries y/o restauraciones coronarias, utilizando fresas redondas tipo Carbide, Endo $Z$ y cucharetas de caries. Además, se irrigó en forma continua con hipoclorito de sodio al 2,5\%, hasta lograr visualizar todas las entradas a los conductos radiculares de la pieza dentaria, para posteriormente sellar con cemento provisorio. 
Los datos fueron recolectados inmediatamente después de realizar el procedimiento clínico. Los datos cualitativos, fueron recolectados a través de entrevistas semi-estructuradas, las que fueron analizadas mediante "análisis de contenido" de acuerdo lo descrito por Bengtsson (2016). De forma inductiva, se definieron códigos, los que luego fueron clasificados en distintas categorías descritas a través de frecuencias absolutas y relativas. Se definieron 10 categorías de acuerdo a lo descrito en la Tabla I. Los datos numéricos fueron recolectados a través de tres escalas de intensidad analógico-visuales, que permitieron conocer la valoración del dolor (Hjermstad et al., 2011). Las escalas utilizadas fueron: la Escala Numérica, en la que el paciente asigna al dolor un valor numérico entre dos puntos extremos (0 a 10); la Escala Visual Análoga (EVA), donde el participante representa su nivel de dolor, en una línea de $10 \mathrm{~cm}$ entre los extremos, ausencia de dolor y dolor insoportable; y la Escala Descriptiva Simple o de Valoración Verbal, en la cual el paciente clasifica su experiencia de acuerdo a una de 4 categorías (ausencia de dolor, dolor leve, dolor moderado o dolor intenso). Se convirtió el resultado de la escala visual análoga, en un valor numérico entre 0 y 10 midiendo la distancia desde el inicio de la línea a la marca realizada por el paciente. Para el análisis de estos datos numéricos, se utilizó el paquete estadístico InfoStat@. Para la escala EVA y la escala numérica se calcularon estadísticos descriptivos de tendencia central (media) y de dispersión (desviación estándar, mínimos y máximos). Para la escala verbal se describieron frecuencias absolutas y relativas.

En términos analíticos, la percepción intraoperatoria fue descrita tanto cualitativamente como cuantitativamente, a modo general y de acuerdo a sexo, arcada y diagnóstico pulpar

\section{RESULTADOS}

Descripción de los participantes. Participaron 20 pacientes, sin embargo, 4 entrevistas fueron excluidas del análisis cualitativo ya que no cumplían con los requisitos mínimos para ser analizadas. Los participantes, fueron en su mayoría de sexo femenino $(n=15,75 \%)$, de edades entre 18 y 78 años y con una media de edad de 46 años. Para el estudio, se seleccionaron tratamientos de molares maxilares $(n=10)$ y mandibulares $(n=10)$, en igual cantidad.
Las piezas tratadas presentaron en su mayoría un diagnóstico pulpar de pulpitis irreversible sintomática $(n=14,70 \%)$, seguido de pulpa normal $(n=3,15 \%)$, necrosis pulpar $(n=2,10 \%)$ y pulpitis irreversible asintomática $(n=1,5 \%)$, de acuerdo a los criterios diagnósticos definidos por Asociación Americana de Endodoncia (AAE Consensus Conference Recommended Diagnostic Terminology, 2009). No se presentaron casos con diagnósticos de pulpitis reversible, los molares con diagnósticos de diente previamente tratado y diente con terapia previamente iniciada fueron excluidos de este estudio.

Las piezas tratadas, presentaron en su mayoría un diagnóstico apical de tejidos periapicales normales ( $n=10,60 \%)$, seguido de periodontitis apical sintomática $(n=5,25 \%)$ y periodontitis apical asintomática ( $n=5,25 \%)$, de acuerdo a los criterios diagnósticos definidos por Asociación Americana de Endodoncia. No se presentaron casos con diagnósticos de absceso apical crónico, ni osteítis condensante.

Los resultados de las escalas cuantitativas para la evaluación del dolor se presentan en la Tabla II. En general, se observaron puntuaciones bajas en cuanto a la valoración del dolor. Al evaluar lo descrito por los participantes, sobre la valoración del dolor intraoperatorio en relación al sexo, se observó que las participantes de sexo femenino, obtuvieron medias mayores que los participantes de sexo masculino en ambas escalas. En relación a la arcada en la cual se ubicó el molar tratado, se observaron medias mayores en el tratamiento de molares mandibulares, que en el tratamiento de molares maxilares en ambas escalas. Finalmente, en relación al diagnóstico pulpar de la pieza a tratar, en ambas escalas se observó que, en dientes con diagnóstico de pulpitis irreversible sintomática, se observaron puntajes medios mayores que en los dientes con diagnóstico de pulpitis irreversible asintomática, pulpa normal y necrosis pulpar.

Al evaluar lo descrito por los participantes, mediante la escala verbal de valoración del dolor intraoperatorio (tabla III), se observó que la mayoría de los participantes percibieron algún grado de dolor durante el tratamiento ( $55 \%$ ), clasificándolo en su mayoría como dolor leve y luego como dolor moderado, ninguno de ellos indicó percibir dolor intenso. Por otro lado, $45 \%$ describió ausencia de dolor durante el procedimiento. La mayoría de los participantes de sexo femenino describieron presentar dolor $(60 \%)$ durante la atención, ya sea leve o moderado. Los pacientes de sexo masculino, describieron en su mayoría au- 
PARIS, L.; FIGUEROA, D.; ALCÁNTARA, R.; LAGOS, G. \& MUÑOZ, F. Percepción intraoperatoria del paciente sobre la preparación de la cavidad de acceso endodontico. Int. J. Odontostomat., 12(3):211-218, 2018.

Tabla I. Categorías construidas a partir de las entrevistas y sus códigos.

\begin{tabular}{|c|c|c|}
\hline Categorias & Códigos & Ejemplo \\
\hline Ansiedad Ante el Tratamiento & $\begin{array}{l}\text { Desconfianza } \\
\text { Miedo } \\
\text { Predisposición a una mala experiencia } \\
\text { Predisposición al dolor } \\
\text { Preocupación } \\
\text { Llamar la atención el ins trumental }\end{array}$ & $\begin{array}{l}\text { "En general, pienso siempre que me puede doler" } \\
\text { Paciente femenino } 69 \text { años }\end{array}$ \\
\hline $\begin{array}{l}\text { Aspectos que Generan } \\
\text { Curiosidad }\end{array}$ & $\begin{array}{l}\text { Llamar la atención los procedimientos } \\
\text { Procedimiento difícil } \\
\text { Sentir olor }\end{array}$ & $\begin{array}{l}\text { "Harto uso de máquinas, de instrumentos" } \\
\text { Paciente femenino } 62 \text { años }\end{array}$ \\
\hline Ausencia de Dolor & $\begin{array}{l}\text { Ver sangre } \\
\text { Ausencia de dolor } \\
\text { Ausencia de molestias } \\
\text { Ausencia de sensación }\end{array}$ & $\begin{array}{l}\text { "No sé, algo me hacía, pero yo no sentía nada" } \\
\text { Paciente masculino } 69 \text { años }\end{array}$ \\
\hline $\begin{array}{l}\text { Experiencia Favorable Durante } \\
\text { el Tratamiento }\end{array}$ & $\begin{array}{l}\text { Buena experiencia } \\
\text { Comodidad } \\
\text { Experiencia normal } \\
\text { Relajación } \\
\text { Tranquilidad }\end{array}$ & "Para mí fue agradable" Paciente masculino 69 años \\
\hline Experiencia Previa & $\begin{array}{l}\text { Antecedentes de experiencia previa } \\
\text { Experiencia previa desfavorable } \\
\text { Sin experiencia previa }\end{array}$ & $\begin{array}{l}\text { "La dife rencia con otras endodoncias que me había } \\
\text { hecho, no, estuvo todo normal" } \\
\text { Paciente femenino } 45 \text { años }\end{array}$ \\
\hline $\begin{array}{l}\text { Incomodidad Durante el } \\
\text { Tratamiento }\end{array}$ & $\begin{array}{l}\text { Dificultad para respirar por aislamiento } \\
\text { Incomodidad con aislamiento } \\
\text { Incomodidad por sillón dental } \\
\text { Sensación de sequedad }\end{array}$ & $\begin{array}{l}\text { "Estaba incómodo en algún momento con todo el } \\
\text { tema de la goma, de la aislación, de la goma en la } \\
\text { boca" } \\
\text { Paciente masculino } 34 \text { años }\end{array}$ \\
\hline $\begin{array}{l}\text { Percepcion de la Atencion } \\
\text { Profesional }\end{array}$ & $\begin{array}{l}\text { Buen trabajo profesional } \\
\text { Percepción favorable de la atención } \\
\text { Sentir seguridad }\end{array}$ & $\begin{array}{l}\text { "Sentí que la atención estaba buena" } \\
\text { Paciente femenino } 69 \text { años }\end{array}$ \\
\hline $\begin{array}{l}\text { Percepcion de la Duracion del } \\
\text { Tratamiento }\end{array}$ & $\begin{array}{l}\text { Procedimiento de corta duración } \\
\text { Procedimiento de larga duración }\end{array}$ & $\begin{array}{l}\text { "Es largo, largo el proceso" Paciente femenino } 62 \\
\text { años }\end{array}$ \\
\hline $\begin{array}{l}\text { Presencia de Dolor en algun } \\
\text { Grado }\end{array}$ & $\begin{array}{l}\text { Sentir dolor } \\
\text { Sentir molestias } \\
\text { Sentir punción }\end{array}$ & $\begin{array}{l}\text { "De primera me dolió un poquito" } \\
\text { Paciente femenino } 24 \text { años }\end{array}$ \\
\hline Sensacion Anestesica & $\begin{array}{l}\text { Tener sensación inespecífica } \\
\text { Aneste sia efectiva } \\
\text { Anestesia no efectiva }\end{array}$ & $\begin{array}{l}\text { "Con la anestesia no sentí nada" } \\
\text { Paciente femenino } 50 \text { años }\end{array}$ \\
\hline
\end{tabular}

sencia de dolor, seguido por percepción de dolor leve durante el tratamiento. Al medir la valoración del dolor intraoperatorio según arcada en la escala verbal, en la mayoría de los tratamientos realizados en molares mandibulares, los participantes declararon percibir dolor (70 \%), en su mayoría leve y en, algunos casos, moderado. En cambio, en la mayor parte de los tratamientos realizados en molares maxilares, los participantes declararon ausencia de dolor y sólo un $40 \%$ declaró haber presentado dolor ya sea leve o moderado. Al hacer esta descripción de acuerdo al diagnóstico, todos los participantes con molares cuyo diagnóstico fue pulpa normal y necrosis pulpar, describieron ausencia de dolor durante la atención. Por otro lado, en los molares cuyo diagnóstico fue pulpitis irreversi- ble, un $71,4 \%$ de los participantes, describió percibir dolor ya sea leve o moderado. Sólo un participante, tuvo un molar con diagnóstico de pulpitis irreversible asintomática, quien indicó percibir dolor leve durante la atención.

Se analizó el contenido del discurso recogido en las entrevistas a los participantes y desde él, se construyeron categorías emergentes, las que fueron descritas de acuerdo a los porcentajes de "referencia" $(n=149)$ o de "menciones" realizadas. Se observó un mayor porcentaje de referencias bajo la categoría "Ansiedad ante el tratamiento", seguida de "Percepción de la atención profesional". Sin embargo, las referencias al dolor en su totalidad (25\%) ya sea "Ausencia 
PARIS, L.; FIGUEROA, D.; ALCÁNTARA, R.; LAGOS, G. \& MUÑOZ, F. Percepción intraoperatoria del paciente sobre la preparación de la cavidad de acceso endodontico. Int. J. Odontostomat., 12(3):211-218, 2018.

Tabla II. Estadísticos descriptivos para las escalas cuantitativas de valoración del dolor. $\mathrm{n}=20$

\begin{tabular}{|c|c|c|c|c|c|c|c|}
\hline Escalas Valoracion Dolor & Categorías & & $\mathrm{n}$ & Media & D.E. & Mín. & Máx. \\
\hline \multirow[t]{9}{*}{ Escala Númerica } & General & & 20 & 1,75 & 1,89 & 0,00 & 6,00 \\
\hline & Sexo & Femenino & 15 & 2,0 & 2,0 & 0,0 & 6,0 \\
\hline & & Masculino & 5 & 1,0 & 1,4 & 0,0 & 3,0 \\
\hline & Arcada & Mandibular & 10 & 2,1 & 1,66 & 0 & 5 \\
\hline & & Maxilar & 10 & 1,4 & 2,12 & 0 & 6 \\
\hline & Diagnóstico pulpar & Pulpa normal & 3 & 0,67 & 1,15 & 0 & 2 \\
\hline & & P.I. Sintomática & 14 & 2,21 & 2,01 & 0 & 6 \\
\hline & & P.I. Asintomática & 1 & 2 & 0 & 2 & 2 \\
\hline & & Necrosis Pulpar & 2 & 0 & 0 & 0 & 0 \\
\hline \multirow[t]{9}{*}{ Escala EVA } & General & & 20 & 1,50 & 1,60 & 0,00 & 5,40 \\
\hline & Sexo & Femenino & 15 & 1,7 & 1,7 & 0,3 & 5,4 \\
\hline & & Masculino & 5 & 1,0 & 1,1 & 0,0 & 2,2 \\
\hline & Arcada & Mandibular & 10 & 1,75 & 1,54 & 0,2 & 4,5 \\
\hline & & Maxilar & 10 & 1,23 & 1,68 & 0 & 5,4 \\
\hline & Diagnóstico pulpar & Pulpa normal & 3 & 0,53 & 0,25 & 0,3 & 0,8 \\
\hline & & P.I. Sintomática & 14 & 1,87 & 1,77 & 0 & 5,4 \\
\hline & & P.I. Asintomática & 1 & 1,2 & 0 & 1,2 & 1,2 \\
\hline & & Necrosis Pulpar & 2 & 0,4 & 0,14 & 0,3 & 0,5 \\
\hline
\end{tabular}

de dolor" o "Presencia de dolor en algún grado" también destacaron (Fig. 1). Al analizar el porcentaje de referencias realizadas a cada categoría por sexo, se observó para los participantes de sexo femenino $(n=107)$ un mayor porcentaje de referencias en la categoría "Percepción de la atención profesional" (16,8 $\%)$, seguido por "Ansiedad ante el tratamiento" (15\%) y "Presencia de dolor en algún grado" (15\%). Para los participantes de sexo masculino $(n=42)$, se observó un mayor porcentaje de referencias a la categoría "Ansiedad ante el tratamiento" (21,4\%), seguido por "Ausencia de dolor" (16,7 \%) y "Experiencia favorable durante el tratamiento" (16,7\%). Al analizar el porcentaje de referencias de los participantes, en relación a la ubicación del molar en la arcada y categoría, se observó que en los casos en que se trataron molares mandibulares $(n=100)$ la mayoría de las referencias recayeron en las categorías: "Percepción de la atención profesional" (19\%), "Ansiedad ante el tratamiento" (18\%), "Presencia de dolor en algún grado" (13\%) y "Experiencia favorable durante el tratamiento" (12 $\%)$. Por otro lado, en los casos en que se trataron molares maxilares $(n=49)$, las mayorías de las referencias recayeron en las categorías: "Aspectos que generan curiosidad" (22,4 \%), "Ansiedad ante el tratamiento" (14,3\%), "Presencia de dolor en algún grado" (12,2\%), "Ausencia de dolor" (12,2\%) y "Experiencia favorable durante el tratamiento" (12,2\%). Al analizar el porcentaje de referencias en relación al diagnósti$\mathrm{co}$, para los participantes en los que se realizaron tratamientos con diagnóstico de pulpa normal $(n=22)$, se observó un mayor porcentaje de referencias a las ca- tegorías "Aspectos que generan curiosidad" (22,7\%) y "Experiencia favorable durante el tratamiento" $(22,7$ $\%)$, seguidas por "Ausencia de dolor" (13,6 \%) e "Incomodidad durante el tratamiento" (13,6\%). En el caso de los molares con diagnóstico de pulpitis irreversible sintomática $(n=7)$, se observó un mayor porcentaje de referencias en la categoría "Ansiedad ante el tratamiento" $(17,5 \%)$, seguida por "Percepción de la atención profesional" (15,8\%) y "Presencia de dolor en algún grado" (15,8 \%). En el caso de los molares con diagnóstico de necrosis pulpar $(n=120)$, se observó un mayor porcentaje de referencias a la categoría "Percepción de la atención profesional" (42,9\%), seguido por "Aspectos que generan curiosidad" (28,6 \%).

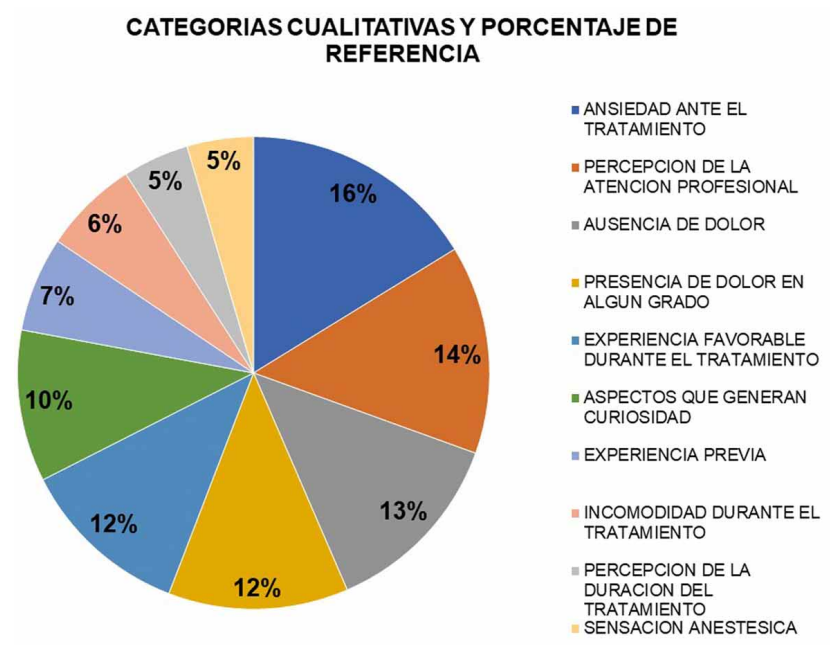

Fig. 1. Porcentaje de referencias de los participantes a las categorías descritas en el análisis cualitativo. 
PARIS, L.; FIGUEROA, D.; ALCÁNTARA, R.; LAGOS, G. \& MUÑOZ, F. Percepción intraoperatoria del paciente sobre la preparación de la cavidad de acceso endodontico. Int. J. Odontostomat., 12(3):211-218, 2018.

Tabla III. Frecuencias absolutas y relativas de los resultados de la escala verbal de valoración del dolor.

\begin{tabular}{|c|c|c|c|c|c|c|c|c|c|c|c|c|c|c|c|c|c|c|}
\hline \multirow{3}{*}{ Escala } & \multirow{2}{*}{\multicolumn{2}{|c|}{ General }} & \multicolumn{4}{|c|}{ Sexo } & \multicolumn{4}{|c|}{ Arcada } & \multicolumn{8}{|c|}{ Diagnóstico Pulpar } \\
\hline & & & \multicolumn{2}{|c|}{$\mathrm{F}$} & \multicolumn{2}{|c|}{ M } & \multicolumn{2}{|c|}{ MAX } & \multicolumn{2}{|c|}{ MAND } & \multicolumn{2}{|c|}{ PN } & \multicolumn{2}{|c|}{ PIS } & \multicolumn{2}{|c|}{ PIA } & \multicolumn{2}{|c|}{ NP } \\
\hline & $\mathrm{n}$ & $\%$ & $\mathrm{n}$ & $\%$ & $\mathrm{n}$ & $\%$ & $\mathrm{n}$ & $\%$ & $\mathrm{n}$ & $\%$ & $\mathrm{n}$ & $\%$ & $\mathrm{n}$ & $\%$ & $\mathrm{n}$ & $\%$ & $n$ & $\%$ \\
\hline Ausencia de Dolor & 9 & 45 & 6 & 40 & 3 & 60 & 6 & 60 & 3 & 30 & 3 & 100 & 4 & 28,6 & 0 & 0 & 2 & 100 \\
\hline Dolor Leve & 8 & 40 & 6 & 40 & 2 & 40 & 2 & 20 & 6 & 60 & 0 & 0 & 7 & 50 & 1 & 100 & 0 & 0 \\
\hline Dolor Moderado & 3 & 15 & 3 & 20 & 0 & 0 & 2 & 20 & 1 & 10 & 0 & 0 & 3 & 21,4 & 0 & 0 & 0 & 0 \\
\hline Dolor Intenso & 0 & 0 & 0 & 0 & 0 & 0 & 0 & 0 & 0 & 0 & 0 & 0 & 0 & 0 & 0 & 0 & 0 & 0 \\
\hline Total & 20 & 100 & 15 & 100 & 5 & 100 & 10 & 100 & 10 & 100 & 3 & 100 & 14 & 100 & 1 & 100 & 2 & 100 \\
\hline
\end{tabular}

F: femenino, M: masculino, MAX: maxilar, MAND: mandibular, PN: pulpa normal, PIS: pulpitis irreversible sintomática, PIA: pulpitis irreversible asintomática, NP: necrosis pulpar

Durante el tratamiento, fue necesario reforzar la anestesia en 7 casos (35\%), 2 (40\%) casos del sexo masculino y 5 (33\%) del femenino. En el $60 \%$ (6) de los tratamientos realizados en molares mandibulares y en el $10 \%$ (1) de los tratamientos realizados en molares maxilares, fue reforzada la anestesia durante la sesión. Por último, se reforzó la anestesia sólo en tratamientos realizados en molares con pulpitis irreversible sintomática, en un $50 \%$ de estos tratamientos.

\section{DISCUSIÓN}

Se realizó una investigación de alcance descriptivo, motivada por la falta de conocimiento científico sobre el dolor experimentado durante el tratamiento de endodoncia. Como una manera de simplificar la evaluación de los datos obtenidos, sólo se contempló la valoración del dolor intraoperatorio en la etapa de acceso endodóntico, considerando que esta etapa también es desarrollada durante la atención de urgencia por el odontólogo general. Para ello, se consideró la evaluación subjetiva del paciente, sobre su experiencia dolorosa, utilizando tanto escalas cuantitativas o de intensidad analógico-visuales como un análisis cualitativo de la experiencia descrita por el sujeto. Las primeras, son consideradas en la literatura como un método válido y confiable para medir el dolor. Por otro lado, el análisis cualitativo, contribuye a una mejor comprensión de la condición humana en diferentes contextos y situaciones. (Serrano-Atero et al.).

Los resultados de este estudio fueron similares a lo descrito por Unruh et al. (1999), en el que las mujeres informaron más dolor que los hombres; y al llevado a cabo por Liddell \& Locker (1997), quienes observaron umbrales de dolor menores en mujeres. Además, coinciden con lo descrito por Segura-Egea et al. (2009), quienes observaron que la mayoría de los hom- bres no experimentaron dolor durante el tratamiento endodóntico, a diferencia de un alto porcentaje de mujeres. Cabe resaltar que los resultados de este estudio pudieran no ser representativos, ya que el número de mujeres y hombres no fue equitativo. Por otro lado, la categoría más frecuente en las entrevistas a las participantes de sexo femenino, fue "Percepción de la atención profesional" seguida por la "Presencia de dolor en algún grado". A raíz de ello se podría pensar que la existencia de dolor intraoperatorio durante el tratamiento puede no ser lo más importante para la paciente de sexo femenino; o, incluso, que pudiese haber existir influencia de otros factores, tales como la atención profesional y la ansiedad en la percepción de dolor intraoperatorio. En el caso de los participantes de sexo masculino, la ausencia de dolor fue la segunda categoría más nombrada, seguida por las referencias a una experiencia favorable. Por ello, es posible presumir que existe cierta relación entre sí, donde la ausencia de dolor contribuiría a una percepción más favorable de la experiencia o viceversa. Por otro lado, la "Ansiedad ante el tratamiento" estuvo también entre lo más nombrado por los participantes de sexo masculino, por ello es posible suponer que la percepción de ansiedad es algo común en pacientes de ambos sexos y no estaría relacionada con la presencia o ausencia de dolor durante el tratamiento. En relación a estos resultados, Watkins et al. describieron que las mujeres sienten mayor ansiedad y preocupación por el dolor que pudieran sentir en el tratamiento endodóntico.

Al analizar los resultados por arcada, estos coinciden con lo reportado por Segura-Egea \& CisnerosCabello, quienes observaron un porcentaje significativamente más alto de dolor leve en dientes mandibulares que en dientes maxilares durante el tratamiento endodóntico. Otros estudios que han abordado estos efectos, han descubierto que la tasa de pacientes que experimentan dolor moderado a severo durante el tratamiento del conducto radicular oscila 
entre 12-35 \% (Kayaoglu et al.). En general, la mayoría de los estudios concluyen que los molares mandibulares cuya pulpa se encuentra inflamada presentan un mayor riesgo de dolor intraoperatorio (Kayaoglu et al.). Por el contrario, en el estudio desarrollado por Martín-Gónzalez et al. (2012), se observó que la arcada dentaria no influía significativamente en el nivel de dolor intraoperatorio durante la terapia endodóntica. En nuestro estudio, la anestesia fue reforzada en un $60 \%$ de los tratamientos realizados en molares mandibulares, lo que coincide con lo descrito por diversos autores, que han reportado la existencia de una inervación accesoria a nivel mandibular que puede ser origen del fracaso anestésico en un $10 \%$ a un $20 \%$ de los casos, si se bloquea el nervio dentario inferior sin refuerzo (González, 2016). En el análisis cualitativo, lo más nombrado por los participantes en relación a la arcada maxilar estuvo dentro de la categoría "Aspectos que generan curiosidad" seguido de "Ansiedad ante el tratamiento". Se puede deducir que esto es consecuente con la menor sensación de dolor intraoperatorio percibida en los dientes maxilares y que la presencia de dolor, aunque en menor grado, puede cobrar mayor relevancia debido a la influencia de la ansiedad previa al tratamiento. En el caso de la arcada mandibular, las categorías más frecuentemente nombradas fueron "Percepción de la atención profesional", "Ansiedad ante el tratamiento" y "Presencia de dolor en algún grado". Este último factor se relaciona directamente con las medidas de dolor intraoperatorio reportadas por los pacientes, además, puede estar influenciado por la ansiedad ante el tratamiento. El reporte de ansiedad fue un común denominador en ambas arcadas y podría influir, ya sea de forma positiva o negativa, en la percepción de la atención profesional por parte del paciente.

En relación al diagnóstico pulpar, se observó que los resultados son similares a lo descrito por Segura-Egea et al., quienes observaron que el tratamiento de endodoncia fue significativamente más doloroso en dientes con pulpitis irreversible que en dientes con pulpas necróticas. En nuestra investigación, en $50 \%$ (7) de los tratamientos realizados en molares con pulpitis irreversible sintomática, fue reforzada la anestesia durante la sesión. Hay varias hipótesis que explican la falla anestésica, sin embargo, ninguna por sí sola explica todas las situaciones clínicas. Dentro de ellas, la química básica señala que la disminución del $\mathrm{pH}$ de los tejidos inflamados, reduce la cantidad de anestesia básica disponible para penetrar en la membrana de un nervio, en consecuencia, hay menos de la forma ionizada dentro del nervio para lograr la anes- tesia (Rosenberg, 2014). También se destaca, que los pacientes con dolor a menudo son aprensivos, lo que reduce su umbral de dolor y favorece a la elaboración de un ciclo negativo, que comienza con aprehensión y deriva a un umbral de dolor disminuido, complicando la anestesia y resultando en un aumento de la aprensión inicial (Rosenberg). Por otro lado, "Ansiedad ante el tratamiento" fue la categoría más nombrada en la entrevista a los pacientes con diagnóstico de pulpitis irreversible sintomática, seguida por "Presencia de dolor en algún grado" y "Percepción de la atención profesional". Este resultado podría avalar la deducción que señala que los pacientes con dolor preoperatorio podrían presentarse ansiosos y aprehensivos a la consulta, lo que disminuiría el umbral doloroso y, por lo tanto, el efecto anestésico no sería el esperado. Si lo comparamos con los otros diagnósticos estudiados, encontramos que, en pacientes con pulpa normal y necrosis pulpar, no se nombra en ninguna ocasión frases referentes a "Presencia de dolor en algún grado".

Conocer la percepción de dolor de cada paciente, a través de un análisis de datos cualitativos, constituye la principal fortaleza y novedad de este estudio. Además, se utilizaron técnicas para la valoración de la percepción de dolor ampliamente validadas en la literatura científica. Sin embargo, la baja representatividad de la muestra, además de la ausencia de estandarización por sexo, impidió valorar adecuadamente dicha variable. Creemos importante, complementar los resultados de este estudio con una evaluación similar en las otras etapas del tratamiento de endodoncia (instrumentación y/o obturación) y/o en tratamientos realizados en una sola sesión. Esto permitiría realizar comparaciones y analizar si la etapa del acceso podría o no ser la más crítica en cuanto a la percepción dolorosa.

\section{CONCLUSIONES}

Podemos concluir que, si bien se constató dolor intraoperatorio durante la etapa de acceso endodóntico, su carácter fue leve en la mayoría de los casos. Esta percepción de dolor, fue mayor en dientes mandibulares al igual que en el diagnóstico de pulpitis irreversible sintomática. A pesar de ello, la presencia de dolor, no fue lo más referido por los participantes durante las entrevistas, en ellas se observó una mayor relevancia de aspectos como la ansiedad ante el tratamiento y la percepción de la atención profesional. 
PARIS, L.; FIGUEROA, D.; ALCÁNTARA, R.; LAGOS, G. \& MUÑOZ, F. Patient intraoperatory perception about endodontic access cavity preparation. Int. J. Odontostomat., 12(3):211-218, 2018.

ABSTRACT: There is a common belief that endodontic treatment is the most painful dental treatment of all. Despite this idea, intraoperative perception during endodontic treatment has not been fully studied. Therefore, the aim of this research was to describe the perception of pain and the intraoperative experience of endodontic treated patients, during the endodontic access cavity preparation. We analyzed the valuations on intraoperative pain completed by 20 patients, treated at the endodontic clinic of a traditional Chilean university, during the endodontic access cavity preparation in molars. For this purpose, quantitative scales of pain intensity assessment and qualitative content analysis of a semi-structured interview were employed. Female participants, treatments in mandibular molars and in teeth diagnosed with symptomatic irreversible pulpitis obtained higher means in terms of assessment of pain intensity. However, in most cases the pain was described as mild. In the qualitative analysis the participant's descriptions were grouped into 11 categories. There was a higher percentage of references to the category "Anxiety before treatment" (16 \%) followed by "Perception of professional care" (14\%). However, references to pain in its totality (25\%) either to "Absence of pain" (13\%) or to "Presence of pain to some degree" (12\%) also stood out. It was concluded that among the participants of this investigation there was perception of intraoperative pain during the endodontic access stage, however, this was mild in most cases.

KEY WORDS: pain, intraoperative, endodontic, mixed methods research.

\section{REFERENCIAS BIBLIOGRÁFICAS}

AAE Consensus Conference Recommended Diagnostic Terminology. J. Endod., 35(12):1634, 2009.

Bengtsson, M. How to plan and perform a qualitative study using content analysis. NursingPlus Open, 2:8-14, 2016.

González, L. Inervación accesoria de dientes mandibulares: Revisión de la literatura. Acta Biocl., 6(11):108-19, 2016.

Hjermstad, M. J.; Fayers, P. M.; Haugen, D. F.; Caraceni, A.; Hanks, G. W.; Loge, J. H.; Fainsinger, R.; Aass, N.; Kaasa, S. \& European Palliative Care Research Collaborative (EPCRC). Studies comparing Numerical Rating Scales, Verbal Rating Scales, and Visual Analogue Scales for assessment of pain intensity in adults: a systematic literature review. J. Pain Symptom Manag, 41(6):1073-93, 2011.

Kayaoglu, G.; Gürel, M.; Saricam, E.; Ilhan, M. N. \& Ilk, O. Predictive model of intraoperative pain during endodontic treatment: prospective observational clinical study. J. Endod., 42(1):36-41, 2016.

Liddell, A. \& Locker, D. Gender and age differences in attitudes to dental pain and dental control. Community Dent. Oral Epidemiol., 25(4):314-8,1997.
Martín-González, J.; Echevarría-Pérez, M.; Sánchez-Domínguez, B.; Tarilonte-Delgado, M. L.; Castellanos-Cosano, L.; López-Frías, F. J. \& Segura-Egea, J. J. Influence of root canal instrumentation and obturation techniques on intra-operative pain during endodontic therapy. Med. Oral Patol. Oral Cir. Bucal, 17(5):e9128, 2012.

Pak, J. G. \& White, S. N. Pain prevalence and severity before, during, and after root canal treatment: a systematic review. J. Endod., 37(4):429-38, 2011.

Randall, C. L.; McNeil, D. W.; Shaffer, J. R.; Crout, R. J.; Weyant, R. J. \& Marazita, M. L. Fear of pain mediates the association between MC1R genotype and dental fear. J. Dent. Res., 95(10):1132-7, 2016.

Rosenberg, P. A. Endodontic pain. Endod.Top., 30(1):75-98, 2014.

Segura-Egea, J. J. \& Cisneros-Cabello, R. Valoración del dolor intraoperatorio experimentado por los pacientes durante el tratamiento endodóncico no quirúrgico. Cient. Dent., 5(1):31-8, 2008.

Segura-Egea, J. J.; Cisneros-Cabello, R.; Llamas-Carreras, J. M. \& Velasco-Ortega, E. Pain associated with root canal treatment. Int. Endod. J., 42(7):614-20, 2009.

Serrano-Atero, M. S.; Caballero, J.; Cañas, A.; García-Saura, P. L.; Serrano-Álvarez, C. \& Prieto, J. Valoración del dolor (I). Rev. Soc. Esp. Dolor, 9(2):94-108, 2002.

Siqueira Jr., J. F. \& Barnett, F. Interappointment pain: mechanisms, diagnosis, and treatment. Endod. Top., 7(1):93-109, 2004.

Thomas, R. How pain is controlled in endodontic therapy. SAAD Dig., 31:32-7, 2015.

Unruh, A. M.; Ritchie, J. \& Merskey, H. Does gender affect appraisal of pain and pain coping strategies? Clin. J. Pain, 15(1):31-40, 1999.

Walton, R. E. \& Torabinejad, M. Managing local anesthesia problems in the endodontic patient. J. Am. Dent. Assoc., 123(5):97-102, 1992.

Watkins, C. A.; Logan, H. L. \& Kirchner, H. L. Anticipated and experienced pain associated with endodontic therapy. J. Am. Dent. Assoc., 133(1):45-54, 2002.

Woolf, C. J. \& Salter, M. W. Neuronal plasticity: increasing the gain in pain. Science, 288(5472):1765-9, 2000.

Dirección para correspondencia

Francisca Muñoz Campos

Cirujano-Dentista, Especialista en Endodoncia

Magister en Educación Médica para Ciencias de la Salud

Profesor Asistente

Departamento de Odontología Restauradora

Facultad de Odontología

Universidad de Concepción

Roosevelt 1550

Casilla 160-c, 4070369

Concepción

CHILE

E-mail: francmunoz@udec.cl

Recibido : 16-05-2018

Aceptado: 14-06-2018 